\title{
Carrier Frequency and Bandwidth Estimation of Cyclostationary Multiband Signals
}

\author{
Deborah Cohen, Liad Pollak, Yonina C. Eldar \\ Technion - Israel Institute of Technology, Haifa, Israel \\ \{debby@tx, liadpollak@t2, yonina@ee\}.technion.ac.il
}

\begin{abstract}
Communication signals are often cyclostationary, that is they have statistical characteristics that vary periodically in time. The cyclic spectrum, a characteristic function of such signals, exhibits spectral peaks at certain locations, called cyclic frequencies. These locations as well as the cyclic spectrum support are defined by the signal parameters, in particular carrier frequency, bandwidth and symbol rate. In this paper, we propose an estimation algorithm that extracts these from the signal cyclic spectrum. This algorithm can be applied to multiband signals, namely signals composed of more than one transmission. Prior to parameter estimation, the number of signals is first estimated. Exploiting the cyclostationarity of communication signals improves the robustness to noise of the parameter estimation. In particular, the proposed algorithm can be used for Cognitive Radios, which traditionally deal with low signal to noise ratios multiband signals, for spectrum sensing purposes by estimating the carrier frequencies and bandwidths. Simulations demonstrate estimation from synthesized and RF Nyquist samples as well as subNyquist samples.
\end{abstract}

\section{INTRODUCTION}

Recently, the traditional and popular signal detection task has been challenged by Cognitive Radio (CR) [1] applications, perceived as a potential solution to the spectrum over-crowdedness, bridging between its scarcity and sparsity. Even though most of the spectrum is already owned, various studies [2], [3] have shown that it is usually significantly underutilized. CRs would allow secondary users to opportunistically use the licensed spectrum when the corresponding primary user (PU) is not active [1]. CR requirements dictate new challenges for its most crucial task: spectrum sensing has to be robust to noise and performed efficiently and in real-time. A CR typically deals with a wideband signal composed of several narrowband transmissions, which is referred to as a multiband model.

Energy detection fails to meet the performance requirements of CRs, which typically deal with low signal to noise ratio (SNR) regimes. Modeling communication signals as cyclostationary rather than as stationary processes can noticeably improve the detection performance since noise is traditionally stationary [4]. Cyclostationary processes have statistical characteristics that vary periodically, arising from the underlying data modulation mechanisms, such as carrier modulation, periodic keying or pulse modulation. The cyclic spectrum, a characteristic function of such processes, exhibits spectral peaks at certain frequency locations called cyclic frequencies, which are determined by the signal parameters, particularly the carrier frequency and symbol rate [4].

In this work, we consider the estimation of the carrier frequencies and bandwidths of each transmission of the multiband input signal from its cyclic spectrum. In particular, this allows us to determine the signal support for spectrum sensing purposes. Cyclostationarity

This work was funded by the European Unions Horizon 2020 research and innovation progremme under grant agreement ERC-BNYQ, and by the Israel Science Foundation under Grant no. 335/14. Deborah Cohen is grateful to the Azrieli Foundation for the award of an Azrieli Fellowship. has been researched extensively [5]. Traditionally, classification algorithms involve learning with a training phase and detection techniques simply compare the cyclic spectrum or cyclic autocorrelation functions to a certain threshold. Our detector, however, is designed to obey certain requirements: (1) carrier frequency and bandwidth estimation rather than simple signal detection; (2) blind detection, namely without knowledge of the carrier frequencies, bandwidths and symbol rates of the transmissions; (3) simultaneous detection of several transmissions; (4) non-learning approach, i.e. with no training phase, for CR purposes.

Single cycle detectors [6], [7], [8] compute the cyclic spectrum at one particular cyclic frequency, a priori known. Dropping the requirement of a priori knowledge of the cyclic frequency of interest, this last approach is extended to the detection of one cyclic frequency that lies on a predefined grid, in [9]. Cyclostationarity induced by the symbol rate alone is considered. The approach of [8] is also extended to multiples cyclic frequencies in [10]. However, only decisions about the presence of a single transmission is allowed, which does not fit the multiband model. In [11], the authors extend cyclostationary detection to the multiband case, by dividing the wideband channel into predefined narrow subbands. They derive the optimal thresholds for all subbands to allow for efficient use of the unexploited spectrum by the CR without causing harmful interference to the PUs. However, only one sample per subband, which defines a PU, is considered and it is not clear how the cyclic features relate to the signal parameters, such as carrier frequencies and bandwidths.

However, in the above works, no parameter estimation is performed. In [12], the carrier frequency of the input signal is assumed to be known and its symbol rate is estimated using the wavelet transform to locate transients produced from phase changes. This technique is used in [13] for modulation classification purposes. Joint carrier frequency, modulation type and bit rate estimation is performed in [14] using the phase derivative. However, the simulations are carried out at very high SNR regimes, between 35 and $70 \mathrm{~dB}$. These parametric methods consider one single input signal and cannot inherently be extended to multiple signals to fit the multiband model.

An alternative approach considers machine learning tools for the modulation classification of a single signal with unknown carrier frequency and symbol rate. In [15], a peak search and save algorithm is proposed, that includes a training phase where a lookup table containing the locations and number of peaks is built for every potential PU. In [16], the classification is performed using neural networks. A hidden Markov model (HMM) approach is considered in [17]. By combining an HMM and a support vector machine (SVM) classifier, the authors in [18] improve the classification performance. The cyclostationary frequencies, namely carrier and symbol rate, are a byproduct of the classification process. However, these approaches are only suitable for a signal and require a training phase, which would 
be a main drawback for CR purposes. In particular, these techniques can only cope with PUs whose modulation type and parameters were part of the training phase.

In order to overcome the sampling rate bottleneck of wideband signals, cyclostationary detection from sub-Nyquist samples has recently been considered [19], [20], [21]. Different cyclic spectrum reconstruction approaches from sub-Nyquist samples are presented and multi-cycle detectors are applied on the reconstructed cyclic spectrum. Our algorithm can be applied to either of these reconstructed spectra in order to extract the carrier frequencies and bandwidths. In the simulations, we consider the approach of [21].

In this work, we propose a simple carrier frequency and bandwidth estimation from the cyclic spectrum of multiband signals. This approach allows the estimation of several carriers and several bandwidths simultaneously. It does not involve any training and simulations show that it achieves high precision even at low SNR regimes, down to $-17 \mathrm{~dB}$. Furthermore, as we show, our approach can operate on both Nyquist and sub-Nyquist samples, as opposed to certain techniques that necessitate oversampling. We adopt a clustering method; the spectral peaks are detected by simple thresholding and then clustered. The number of signals is estimated from the clusters number and for each transmission, its carrier frequency and bandwidth are estimated from the corresponding clusters. Our main contribution is a simple algorithm that fits the multiband model, without any prior knowledge nor learning requirements. In the simulations, we demonstrate parameter estimation from synthesized Nyquist samples as well as samples obtained from a radio frequency (RF) signal generated by a Universal Software Radio Peripheral (USRP), and subNyquist samples obtained using the Modulated Wideband Converter (MWC) [23].

This paper is organized as follows. Section II presents the cyclostationary multiband model. In Section III, we describe the parameter estimation algorithm from the signal cyclic spectrum. Numerical experiments showing carrier frequency and bandwidth recovery are presented in Section IV.

\section{Cyclostationary Multiband Signals}

\section{A. Multiband Model}

Let $x(t)$ be a real-valued continuous-time signal, supported on $\mathcal{F}=\left[-1 / 2 T_{\mathrm{Nyq}},+1 / 2 T_{\mathrm{Nyq}}\right]$ and composed of up to $N_{\text {sig }}$ uncorrelated cyclostationary transmissions, such that

$$
x(t)=\sum_{i=1}^{N_{\text {sig }}} s_{i}(t) .
$$

Here $s_{i}(t)$ is a zero-mean cyclostationary bandpass process, as defined below, from the class of pulse-amplitude modulation (PAM) signals, such that

$$
\begin{aligned}
s_{i}(t)=\sqrt{2} \cos \left(2 \pi f_{i} t\right) & \sum_{k} a_{k}^{I} g\left(t-k T_{i}\right) \\
& -\sqrt{2} \sin \left(2 \pi f_{i} t\right) \sum_{k} a_{k}^{Q} g\left(t-k T_{i}\right) .
\end{aligned}
$$

The symbols modulating the in-phase and quadrate components are denoted $\left\{a_{k}^{I}\right\}$ and $\left\{a_{k}^{Q}\right\}$, respectively, and $g(t)$ is the pulse shape function. The single-sided bandwidth, the carrier frequency and the symbol period are denoted by $B_{i}, f_{i}$ and $T_{i}$, respectively. Special cases of passband PAM include phase-shift keying (PSK), amplitude and phase modulation (AM-PM) and quadrature amplitude modulation (QAM) [24].

Formally, the Fourier transform of $x(t)$ defined by

$$
X(f)=\lim _{T \rightarrow \infty} \frac{1}{\sqrt{T}} \int_{-T / 2}^{T / 2} x(t) e^{-j 2 \pi f t} \mathrm{~d} t
$$

is zero for every $f \notin \mathcal{F}$. We denote by $f_{\mathrm{Nyq}}=1 / T_{\mathrm{Nyq}}$ the Nyquist rate of $x(t)$. The number of transmissions $N_{\text {sig }}$, their carrier frequencies, bandwidths, symbol rates and modulations, including the symbols $\left\{a_{k}\right\}$ and the pulse shape functions $g(t)$, are unknown, namely the cyclic spectrum reconstruction is performed in a blind scenario.

\section{B. Cyclostationarity}

A process $x(t)$ is said to be cyclostationary with period $T_{0}$ in the wide sense if its mean $\mathbb{E}[x(t)]=\mu_{x}(t)$ and autocorrelation $\mathbb{E}[x(t) x(t+\tau)]=R_{x}(t, \tau)$ are both periodic with period $T_{0}$ [5]:

$$
\mu_{x}\left(t+T_{0}\right)=\mu_{x}(t), \quad R_{x}\left(t+T_{0}, \tau\right)=R_{x}(t, \tau) .
$$

Given a wide-sense cyclostationary random process, its autocorrelation $R_{x}(t, \tau)$ can be expanded in a Fourier series

$$
R_{x}(t, \tau)=\sum_{\alpha} R_{x}^{\alpha}(\tau) e^{j 2 \pi \alpha t},
$$

where $\alpha=m / T_{0}, m \in \mathbb{Z}$ and the Fourier coefficients $R_{x}^{\alpha}$ are referred to as cyclic autocorrelation functions. The cyclic spectrum is obtained by taking the Fourier transform of $R_{x}^{\alpha}$ with respect to $\tau$, namely

$$
S_{x}^{\alpha}(f)=\int_{-\infty}^{\infty} R_{x}^{\alpha}(\tau) e^{-j 2 \pi f \tau} \mathrm{d} \tau,
$$

where $\alpha$ is referred to as the cyclic frequency and $f$ is the angular frequency [5]. If there is more than one fundamental frequency $T_{0}$, then the process $x(t)$ is said to be polycyclostationary in the wide sense. In this case, the cyclic spectrum contains harmonics (integer multiples) of each of the fundamental cyclic frequencies [4].

Another interpretation of the cyclic spectrum we will exploit, is that it is the cross-spectral density $S_{x}^{\alpha}(f)=S_{u v}(f)$ of two frequency-shifted versions of $x(t), u(t) \triangleq x(t) e^{-j \pi \alpha t}$ and $v(t) \triangleq$ $x(t) e^{+j \pi \alpha t}$. Then, from [25], it holds that

$$
S_{x}^{\alpha}(f)=S_{u v}(f)=\mathbb{E}\left[X\left(f+\frac{\alpha}{2}\right) X^{*}\left(f-\frac{\alpha}{2}\right)\right] .
$$

It can be shown [4] that stationary noise $w(t)$ exhibits no cyclic correlation, that is $S_{w}^{\alpha}(f)=0, \quad \alpha \neq 0$. This property is the motivation for cyclostationary detection, in low SNR regimes in particular.

\section{Cyclic Spectrum of Multiband Signals}

Let us denote by $\left[f_{i}^{(1)}, f_{i}^{(2)}\right]$ the right-side support of the $i$ th transmission $s_{i}(t)$. It holds that $B_{i}=f_{i}^{(2)}-f_{i}^{(1)}$ and $f_{i}=\left(f_{i}^{(1)}+\right.$ $\left.f_{i}^{(2)}\right) / 2$. The support region in the $(f, \alpha)$ plane of the cyclic spectrum $S_{s_{i}}^{\alpha}(f)$ of such a bandpass cyclostationary signal is composed of four diamonds, as shown in Fig. 1. More precisely, it holds that [4]

$$
S_{s_{i}}^{\alpha}(f)=0, \quad \text { for }|| f\left|-\frac{|\alpha|}{2}\right| \leq f_{i}^{(1)} \text { or }|f|+\frac{|\alpha|}{2} \geq f_{i}^{(2)} .
$$

Since the transmissions $s_{i}(t)$ are assumed to be zero-mean and uncorrelated (coming from different sources), the cyclic spectrum of $x(t)$ does not contain any additional harmonics which would result from correlation between different transmissions. Therefore, 


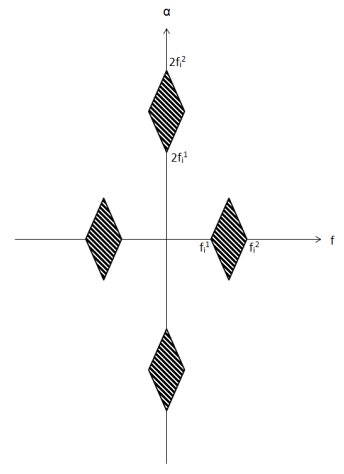

Fig. 1. Support region of the cyclic spectrum of $S_{s_{i}}^{\alpha}(f)$.

the support of $S_{x}^{\alpha}(f)$ is composed of $4 N_{\text {sig }}$ diamonds, namely four diamonds for each transmission as those shown in Fig. 1. Moreover, since $x(t)$ is bandlimited to $\mathcal{F}$, it holds that [4]

$$
S_{x}^{\alpha}(f)=0, \quad \text { for } \quad|f|+\frac{|\alpha|}{2} \geq \frac{f_{\mathrm{Nyq}}}{2} .
$$

Applying (7) for $\alpha= \pm 2 f_{i}$, namely

$$
S_{x}^{ \pm 2 f_{i}}(f)=\mathbb{E}\left[X\left(f+f_{i}\right) X^{*}\left(f-f_{i}\right)\right],
$$

computes the correlation between the positive and negative bands of the $i$ th transmission. This generates a peak, or cyclic feature, in $S_{x}^{\alpha}(f)$ at the location $\left(f=0, \alpha= \pm 2 f_{i}\right)$. By detecting the peak location, we can estimate the carrier of the corresponding transmission $s_{i}(t)$. Furthermore, from (8), it is clear that the occupied bandwidth in the cyclic spectrum at $\alpha= \pm 2 f_{i}$ is equal to the bandwidth $B_{i}$. This will be exploited to estimate the transmission bandwidth.

\section{Goal}

Our objective is to estimate the number of transmissions $s_{i}(t), 1 \leq i \leq N_{\text {sig }}$ present in $x(t)$, their carrier frequencies $f_{i}$ and their bandwidths $B_{i}$. These completely define the signal support, for detection purposes. To that end, we propose to extract the carrier frequencies and the bandwidths from the cyclic spectrum. We note that the transmissions symbol rate can be derived from their bandwidth, as

$$
T_{i}=\frac{1+\gamma}{2 B_{i}},
$$

where $\gamma$ is the excess-bandwidth parameter of $g(t)$ [24]. Therefore, we will focus only on carrier frequency and bandwidth estimation, since the symbol rate can be directly determined from the estimated bandwidth, if the excess-bandwidth parameter $\gamma$ is a priori known.

\section{Parameter Estimation Algorithm}

The proposed estimation algorithm can be decomposed into the following main five steps: cyclic spectrum estimation, preprocessing, thresholding, clustering, parameter estimation. The main contribution of this approach is the estimation of the number of signals, and for each transmission, the estimation of its carrier frequency and bandwidth from the clusters.

\section{A. Cyclic Spectrum Estimation}

The cylic spectrum of the input signal $x(t)$, namely $S_{x}^{\alpha}(f)$ is first computed using (7). In practice, in order to estimate the cyclic spectrum $S_{x}^{\alpha}(f)$ we first compute $X(f)$, using the fast Fourier transform (FFT) on the samples $x[n]$ over a finite time window of size $N_{\text {samp. }}$ The cyclic spectrum is then estimated by averaging over $P$ frames as follows

$$
\hat{S}_{x}^{\alpha}(f)=\sum_{p=1}^{P}\left[X\left(f+\frac{\alpha}{2}\right) X^{*}\left(f-\frac{\alpha}{2}\right)\right] .
$$

The total number of samples is therefore $N_{\text {samp }} P$.

\section{B. Preprocessing}

The preprocessing simply aims at compensating for the presence of stationary noise at the cyclic frequency $\alpha=0$. This is motivated by the fact that the threshold which will be used for peak detection in the next step is proportional to the mean energy of the signal. In low SNR regimes, the energy in DC due to the noise might lead to a very high threshold, annihilating peaks at other cyclic frequencies.

\section{Thresholding}

We can now apply thresholding to the resulting cyclic spectrum in order to find its peaks. The threshold is a design parameter that is set with respect to the average sample energy. For each cyclic frequency $\alpha$, we retain the highest peak along the abscissa axis.

\section{Clustering}

The locations and values of the peaks are then clustered to find the corresponding cyclic feature. A cyclic feature yields a cluster of peaks, rather than a single one, due to the finite sensing time.

1) Clusters number estimation: We start by estimating the number of clusters, which is traditionally required in clustering methods. To that end, we use the elbow method, which can be traced to speculation by Thorndike [26]. This iterative method is based on the ratio between group variance (sum of the variances in each cluster) to the total variance, or variance percentage. More precisely, in each iteration, the number of clusters is increased and clustering is performed with respect to it, as described in the next section. When the ratio between the variance percentage of two consecutive values of the number of clusters becomes higher than a certain threshold, the algorithm stops and the number of clusters is set to its current value. Specifically, we start with 3 clusters and consider only odd numbers of clusters (the peaks all come in pairs due to the symmetry of the cyclic spectrum except for the extra DC peak). The threshold is a design parameter.

2) Clusters separation: Clustering is performed using the kmeans method. Since the cylic spectrum is symmetric with respect to the abscissa axis, we feed the k-means with the sum of the values of the positive cyclic frequencies and their negative counterpart. The kmeans algorithm, which involves a random factor in the initialization, is run several times, and the median result is selected. Each cluster represents a cyclic feature. It follows that, apart from the cluster present in DC which we will remove, the number of signals $N_{\text {sig }}$ is equal to half the number of clusters (when we duplicate the clusters back to the positive and negative cyclic frequencies). We note that the peaks present in the diamonds around $\alpha=0$ (see Fig. 1) are absorbed in the DC cluster for all the transmissions, along with the stationary noise. Thus, the parameter estimation described in the next section only lies on the upper and lower diamonds features (see Fig. 1). 

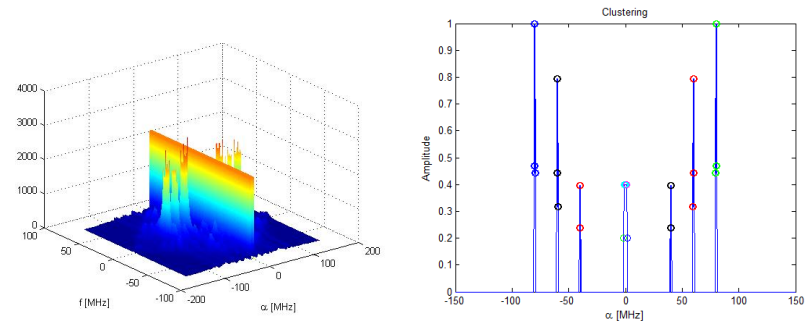

Fig. 2. Cyclic spectrum (left), cyclic peaks clustering (right)

\section{E. Parameter Estimation}

We can now estimate the carrier frequencies and bandwidths of the $N_{\text {sig }}$ transmissions. We first estimate the carrier frequency $f_{i}$ of each transmission. The carrier frequency yields the highest correlation [4] and thus the highest peak, at the cyclic frequency equal to twice its value, namely $\alpha=2 f_{i}$. It is therefore estimated as half the cyclic frequency of the highest peak within the clusters belonging to the same signal.

Last, the bandwidth of each transmission is determined based on its estimated carrier frequency. Again, exploiting the fact that the cylic spectrum is symmetric with respect to the abscissa axis, we compute the sum of its values at $\alpha=2 f_{i}$ and $\alpha=-2 f_{i}$ along the angular frequencies $f$. The bandwidth is found by locating the edge of the support of the angular frequencies. To that end, we choose a threshold, which is a design parameter, and find the farthest location from $f=0$ along $\alpha= \pm 2 f_{i}$ for which the value of the cyclic spectrum $S_{x}^{ \pm 2 f_{i}}(f)$ is above that threshold.

\section{Simulation Results}

In this section, we first illustrate our estimation method. We then investigate the impact of noise on the performance of our estimator. We consider synthesized Nyquist and sub-Nyquist samples as well as RF Nyquist samples.

Let $x(t)$ be composed of $N_{\text {sig }}=3$ BPSK transmissions with Nyquist rate $f_{\mathrm{Nyq}}=150 \mathrm{MHz}$ and carrier frequencies $f_{1}=20 \mathrm{MHz}$, $f_{2}=30 \mathrm{MHz}, f_{3}=40 \mathrm{MHz}$, and with bandwidths $B_{1}=B_{2}=$ $B_{3}=0.67 M H z$. The SNR is set to be $-10 d B$. The cyclic spectrum is estimated by averaging over $P=200$ frames, each with $N_{\text {samp }}=$ 1000. Figure 2 shows the estimated cyclic spectrum, $\hat{S}^{\alpha}(f)$, of $x(t)$ after the preprocessing step and the results from the clustering after duplication. The highest peaks, one for each cyclic frequency $\alpha$ which is obtained at the thresholding step, are clustered into 7 groups (twice the number of signals plus the DC cluster). The estimation results for this setting were as follows: $f_{1}=20.08 \mathrm{MHz}, f_{2}=30.04 \mathrm{MHz}$, $f_{3}=39.94 \mathrm{MHz}$ and $B_{1}=0.67 \mathrm{MHz}, B_{2}=0.62 \mathrm{MHz}, B_{3}=$ $0.71 \mathrm{MHz}$.

We now examine our algorithm estimation performance. For lack of space, we only compare carrier frequency estimation performance of our approach with energy detection. Let $x(t)$ be composed of $N_{\text {sig }}=3$ transmissions. Each transmission is a BPSK modulated signal with carrier frequency $f_{i}$ in the interval $[10-50] \mathrm{MHz}$ and bandwidth $B_{i}$ between 0.6 and $1 M H z$. The carrier frequencies and bandwidths values are drawn uniformly at random for each realisation. The SNR is set to $-10 d B$. The signal $x(t)$ is sampled at the rate $f_{s}=150 \mathrm{MHz}$ and its cyclic spectrum is estimated by averaging over $P=500$ frames, each with $N_{\text {samp }}=1001$. Each experiment is repeated over 1000 realisations. Figure 3 shows the probability of

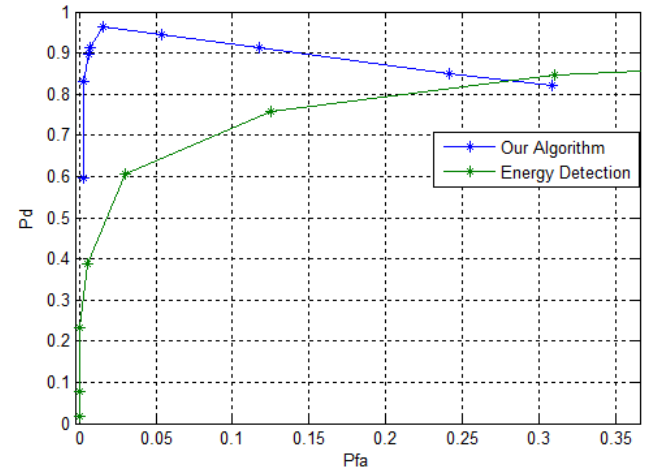

Fig. 3. Receiver operating characteristic (ROC).

detection of the estimator as a function the probability of false alarm. A success is declared when the error of the estimator is below 5 times the frequency resolution, which is is $0.3 \mathrm{MHz}$ in this setting. The probability of detection is computed by averaging over the 3 transmissions. A false alarm is declared if at least one detected signal is in fact only noise. We observe that the probability of detection increases with the probability of false alarm up to a certain point and then decreases. This unusual behavior follows from the fact that the thresholding comes before clustering and other processing tasks. High thresholds lead to few peaks, which in turn yields low probabilities of detection and false alarm. When the threshold descreases, both probabilities increase with the detection improving faster. Last, when the threshold gets too low, several clusters belonging to different signals can merge, affecting the probability of detection, while the probability of false alarm obviously increases.

Our algorithm has been tested on samples from an RF signal generated by a NI ${ }^{\circledR}$ USRP-2942-R RF generator. Here, $x(t)$ is composed of $N_{\text {sig }}=2$ transmissions with carriers $f_{1}=38 \mathrm{MHz}$ and $f_{2}=47 \mathrm{MHz}$, and bandwidths $B_{1}=B_{2}=0.25 \mathrm{MHz}$. The SNR is $-15 d B$, the sampling rate $f_{s}=120 \mathrm{MHz}$, and the frequency resolution $12 \mathrm{KHz}$. The cyclic spectrum is estimated by averaging over $P=992$ frames, each with $N_{\text {samp }}=1001$, with overlapping. The estimation results for this setting were as follows: $f_{1}=37.88 \mathrm{MHz}, f_{2}=46.87 \mathrm{MHz}$, and $B_{1}=0.22 \mathrm{MHz}$, $B_{2}=0.36 \mathrm{MHz}$.

Last, we applied our algorithm to a cyclic spectrum reconstructed from sub-Nyquist samples obtained using the MWC [23], using the sub-Nyquist cyclic spectrum recovery algorithm from [21] to which we apply our parameter estimation algorithm. Here, $x(t)$ is composed of $N_{\text {sig }}=3$ BPSK transmissions with Nyquist rate $f_{\mathrm{Nyq}}=6.381 \mathrm{GHz}$ and carrier frequencies $f_{1}=1619.06 \mathrm{MHz}$, $f_{2}=1714.30 \mathrm{MHz}, f_{3}=2285.73 \mathrm{MHz}$, and bandwidths $B_{1}=$ $B_{2}=B_{3}=10 \mathrm{MHz}$. The SNR is $10 \mathrm{~dB}$, the sampling rate $f_{s}=2.381 \mathrm{GHz}$, and the frequency resolution $1.587 \mathrm{MHz}$. The cyclic spectrum is estimated by averaging over $P=100$ frames, each with $N_{\text {samp }}=7437$. The estimation results for this setting were as follows: $f_{1}=1619.46 \mathrm{MHz}, f_{2}=1714.73 \mathrm{MHz}, f_{3}=2286.30$, and $B_{1}=9.1 \mathrm{MHz}, B_{2}=9.0 \mathrm{MHz}$ and $B_{3}=9.7 \mathrm{MHz}$.

\section{ACKNOWLEDGEMENT}

The authors would like to thank Prof. Lihi Zelnik-Manor for her valuable input on clustering methods. 


\section{REFERENCES}

[1] J. Mitola, "Software radios: Survey, critical evaluation and future directions," IEEE Aerosp. Electron. Syst. Mag, vol. 8, pp. 25-36, Apr. 1993

[2] FCC, "Spectrum policy task force report: Federal communications commission, tech. rep. 02-135. [online]," http://www.gov/edocs_public/attachmatch/DOC228542Al.pdf, Nov. 2002.

[3] M. McHenry, "NSF spectrum occupancy measurements project summary. shared spectrum co., tech. rep. [online]," http://www.sharedspectrum.com, Aug. 2005.

[4] W. Gardner, Statistical spectral analysis: a non probabilistic theory. Prentice Hall, 1988.

[5] W. A. Gardner, A. Napolitano, and L. Paura, "Cyclostationarity: Half a century of research," pp. 639-697, Apr. 2006.

[6] P. S. Aparna and M. Jayasheela, "Cyclostationary feature detection in cognitive radio using different modulation schemes," Int. Journal of Computer Applications, vol. 47, pp. 12-16, Jun. 2012.

[7] S. Y. Kumar and M. Z. A. Khan, "Robust, block based cyclostationary detection," Int. Conf. on Telecommunications (ICT), pp. 1-5, May 2013.

[8] A. V. Dandawate and G. B. Giannakis, "Statistical tests for presence of cyclostationarity," IEEE Trans. on Signal Processing, pp. 2355-2369, 1994

[9] M. Ghozzi, F. Marx, M. Dohler, and J. Palicot, "Cyclostatilonaritybased test for detection of vacant frequency bands," Int. ICST Conf. on Cognitive Radio Oriented Wireless Networks and Communications (CROWNCOM), pp. 1-5, Jun. 2006.

[10] J. Lunden, V. A. V. Koivunen, and V. H. Poor, "Spectrum sensing in cognitive radios based on multiple cyclic frequencies," Int. ICST Conf. on Cognitive Radio Oriented Wireless Networks and Communications (CROWNCOM), pp. 37-43, Aug. 2007.

[11] S. Tariq, A. Ghafoor, and S. Z. Farooq, "Convexity analysis for cyclostationary detection in cognitive radios," IEEE Symposium on Computers and Commun. (ISCC), Jul. 2012.

[12] Y. T. Chan, J. W. Plews, and K. C. Ho, "Symbol rate estimation by the wavelet transform," IEEE Int. Symposium on Circuits and System (ISCAS), pp. 177-180, Jun. 1997.

[13] D. C. E. Rebeiz, "Blind modulation classification based on spectral correlation and its robustness to timing mismatch," Military Communications Conf. (MILCOM), pp. 277-282, 2011.

[14] R. J. Mammone, R. J. Rothaker, C. I. Podilchuk, S. Davidovici, and D. L. Schilling, "Estimation of carrier frequency, modulation type and bit rate of an unknown modulated signal," IEEE Int. Conf. on Communications (ICC), pp. 1006-1012, 1987.

[15] A. Badawy and T. Khattab, "Robust, block based cyclostationary detection," IEEE Wireless Commun. and Networking Conf. (WCNC), pp. 253-258, Apr. 2014.

[16] A. Fehske, J. Gaeddert, and J. H. Reed, "A new approach to signal classification using spectral correlation and neural networks," IEEE International Symposium on New Frontiers in Dynamic Spectrum Access Networks, pp. 144-150, Nov. 2005.

[17] Aparna P.S and M. Jayasheela, "Cyclostationary feature detection in cognitive radio using different modulation schemes," Int. Journal of Computer Applications, vol. 47, pp. 12-16, June 2012.

[18] X. He, Z. Zeng, and C. Guo, "Signal classification based on cyclostationary spectral analysis and HMM/SVM in cognitive radio," Int. Conf. on Measuring Technology and Mechatronics Automation, pp. 309-312, 2009

[19] Z. Tian, Y. Tafesse, and B. M. Sadler, "Cyclic feature detection with sub-Nyquist sampling for wideband spectrum sensing," IEEE Journal of selected topics in signal processing, 2012.

[20] S. A. Razavi, M. Valkama, and D. Cabric, "High-resolution cyclic spectrum reconstruction from sub-Nyquist samples," IEEE SPAWC, pp 250-254, Jun. 2013.

[21] D. Cohen and Y. C. Eldar, "Cyclic spectrum reconstruction from subNyquist samples," IEEE GLOBECOM, pp. 3132-3137, Dec. 2014.
[22] S. P. N. Shetty and P. Pawelczak, "Identifying spectrum usage by unknown systems using experiments in machine learning," IEEE Wireless Commun. and Networking Conf. (WCNC), pp. 1-6, Apr. 2009.

[23] M. Mishali and Y. C. Eldar, "From theory to practice: Sub-Nyquist sampling of sparse wideband analog signals," IEEE J. Sel. Topics Signal Process., vol. 4, no. 2, pp. 375-391, Apr. 2010.

[24] J. R. Barry, E. A. Lee, and D. G. Messerschmitt, Digital Communication. Springer, 2003.

[25] A. Papoulis, Probability, Random Variables, and Stochastic Processes. McGraw Hill, 1991.

[26] R. L. Thorndike, "Who belong in the family?" Psychometrika, pp. 267276, Dec. 1953. 